\title{
Predictive value of Smac, VEGF and Ki-67 in rectal cancer treated with neoadjuvant therapy
}

\author{
HONGJIANG YAN ${ }^{1}$, RENBEN WANG ${ }^{1}$, JINMING YU $^{1}$, SHUMEI JIANG ${ }^{1}$, \\ $\mathrm{KUNLI}_{\mathrm{ZHU}}{ }^{1}$, DIANBIN MU ${ }^{2}$ and ZHONGFA XU ${ }^{3}$
}

Departments of ${ }^{1}$ Radiation Oncology, ${ }^{2}$ Pathology, and ${ }^{3}$ General Surgery, Shandong Tumor Hospital, Jinan 250117, P.R. China

Received March 24, 2010; Accepted May 27, 2010

DOI: 10.3892/ol_00000113

\begin{abstract}
The present study aimed to identify whether second mitochondria-derived activator of caspase (Smac), vascular endothelial growth factor (VEGF) and proliferating cell nuclear antigen (Ki-67) expression in pre-treatment tumor biopsies are useful predictive markers of tumor response in patients with rectal cancer undergoing pre-operative chemoradiotherapy (CRT). Paraffin-embedded tissues obtained before and after therapy were evaluated by immunohistochemical staining for Smac, VEGF and Ki-67. The study evaluated the correlation of Smac, VEGF and Ki-67 immunoreactivity in tumor biopsies before treatment of tumor response to pre-operative CRT. Regarding Smac, patients with a favorable response to neoadjuvant CRT had higher pre-therapy levels $(\mathrm{p}=0.011)$. The level of Smac expression decreased after neoadjuvant therapy $(p=0.044)$. However, VEGF expression was found to be negatively and significantly correlated with a favorable tumor response to neoadjuvant $\mathrm{CRT}(\mathrm{p}=0.010)$. A transient increase in VEGF expression was detected in the resected specimens following neoadjuvant therapy $(\mathrm{p}=0.030)$. In addition, tumors with a low $\mathrm{Ki}-67$ labeling index (Ki-67-LI) expression were found to be more sensitive to neoadjuvant therapy than those with a high expression of Ki-67-LI $(\mathrm{p}=0.034)$. In contrast to VEGF, the Ki-67 expression level decreased after neoadjuvant therapy. Smac, VEGF and Ki-67 expression levels, assessed immunohistochemically from pre-treatment tumor biopsies, may be useful predictive markers of rectal tumor response to pre-operative CRT.
\end{abstract}

\section{Introduction}

Pre-operative chemoradiotherapy (CRT) is the current standard treatment for locally advanced rectal cancer (1-5). Pre-operative

Correspondence to: Dr Renben Wang, Department of Radiation Oncology, Shandong Tumor Hospital, Jinan 250117, P.R. China

E-mail: wangrenben@tom.com

Key words: rectal cancer, second mitochondria-derived activator of caspase, vascular endothelial growth factor, Ki-67, pre-operative chemoradiotherapy
CRT for locally advanced rectal cancer has several potential advantages, including a decrease in tumor volumes, introduction of downstaging, an increase in the possibility of R0 resection, a reduction in radiation-induced toxicity, enhanced probability of anal sphincter preservation by shrinking large distal tumors, a reduction in local recurrence and improvement in survival (6-10). However, the response of individual tumors to adjuvant therapies is not uniform. The majority of patients benefit from pre-operative CRT while a small percentage of the patient population is less likely to respond to this treatment. In order to offer patients individualized therapy, the identification of predictive markers of cancer response to pre-operative CRT are necessary.

The present study investigated three proteins that are known to play a significant role in the growth and development of numerous tumors.

Second mitochondria-derived activator of caspase (Smac) is a novel pro-apoptotic protein first reported by $\mathrm{Du}$ et al in 2000 (11). Under the induction of apoptotic stimuli, such as an anti-cancer drug, DNA damage, exposure to ultraviolet radiation or chemical signals, the pro-Smac proteins, composed of 293 amino acids, can be cut off from their signal peptides to become active Smac proteins that are then released from mitochondria into the cytosol along with cytochrome $\mathrm{c}$ to promote apoptosis. Through the interaction with inhibitors of apoptosis proteins (IAPs), Smac hydrolyzes caspase-3 protein and enhances the catalytic activity of mature caspase- 3 , thereby promoting apoptosis (11).

Various studies have demonstrated that vascular endothelial growth factor (VEGF) is absent in normal colorectal mucosa, but present in carcinomas $(12,13)$. VEGF is associated with mediation to tumor angiogenesis. VEGF is activated in tumor cells by several factors, including tumor suppressor genes, hypoxia and oncogenes (14). The activation of VEGF results in the formation of new vasculature and endothelial cell migration which supports the growth of the tumor and its nutrient requirements (15). In situ hybridization trials showed that the transcription of VEGF mRNA in rectal tumors is up-regulated during the progression from adenoma to carcinoma $(16,17)$. New blood vessels are characterized by increased permeability, causing less efficient delivery of chemotherapeutic agents and a decreased response to radiotherapy $(18,19)$. Anti-VEGF therapy, in combination with chemotherapy and/or radiotherapy for rectal cancer, is a promising approach $(18,20)$. 
Table I. Change in Smac, VEGF and Ki-67 expression before and after neoadjuvant therapy.

\begin{tabular}{|c|c|c|c|c|c|c|}
\hline & \multicolumn{2}{|c|}{ Smac } & \multicolumn{2}{|c|}{ VEGF } & \multicolumn{2}{|c|}{$\mathrm{Ki}-67$} \\
\hline & Low & High & Negative & Positive & Low & High \\
\hline Pre-treatment & 16 & 24 & 17 & 23 & 20 & 20 \\
\hline Post-treatment & 25 & 15 & 8 & 32 & 23 & 17 \\
\hline P-value & \multicolumn{2}{|c|}{0.044} & \multicolumn{2}{|c|}{0.030} & \multicolumn{2}{|c|}{0.501} \\
\hline
\end{tabular}

$\mathrm{Ki}-67$ is associated with regulation of the cell cycle. It is a nuclear protein that is present in the later G1, S, G2 and M phases of the cell cycle, and is thus a good marker of proliferation (21). The Ki-67 labeling index (Ki-67-LI) is likely to be associated with the downstaging of highly proliferative tumors.

By investigating the change in expression of these proteins, it may be possible to predict which patients respond favorably to pre-operative CRT.

\section{Materials and methods}

Patients. A total of 40 patients with rectal adenocarcinoma were enrolled in the present study. Informed written consent was obtained from each patient. The study was approved by the Ethisc Committee of Shandong Tumor Hospital. The patients underwent flexible endoscopy with rectal biopsy, a complete blood count, a biochemical profile and serum CEA level tests. Chest X-ray, abdominal and pelvic computed tomography (CT), magnetic resonance imaging, PET-CT and/or endoscopic ultrasonography were performed to exclude tumor, node and metastasis (TNM) stage I and IV tumors. Patients with abdominal nodal disease or metastases were excluded from the study. The complete blood count, urinalysis, liver-function tests and chest X-ray were all normal in all 40 patients.

Treatment. The patients underwent CT simulation for three-dimensional conformal radiotherapy planning and the three-field technique (one posterior field and two lateral fields). The clinical target volume of the pre-operative radiotherapy consisted of the primary tumor, the mesentery including vascular supply and the perirectal, presacral and internal iliac nodes (up to the $\mathrm{S} 1 / \mathrm{S} 2$ junction). The planned target area was formed by enlarging the area by $10-15 \mathrm{~mm}$ on the basis of the clinical target volume. Pre-operative radiotherapy was delivered in fractions of 2 Gy to reach a dose of 40 Gy at 5 fractions per week. All 40 patients received concurrent chemotherapy $\left(300 \mathrm{mg} / \mathrm{m}^{2} 5-\mathrm{FU}, 200 \mathrm{mg} / \mathrm{m}^{2}\right.$ oxaliplatin and $100 \mathrm{mg} / \mathrm{m}^{2}$ leucovorin) during the first 5 and last 5 days of radiotherapy. The patients received a total mesorectal excision procedure after a lengthy interval of up to 4-6 weeks. Tumor response was classified as complete response (CR, no residual tumor), partial response (PR, tumor volume diminished $>50 \%$ and/or downstaging) and no response (NR) after post-operative pathological analysis of the tumor specimens.

Immunohistochemical assay of Smac, VEGF and Ki-67. Paraffin-embedded tumors from both the biopsy before treatment $(n=40)$ and the tumor tissue from the resection specimen $(\mathrm{n}=40)$ were used to make donor blocks. Sections $(4 \mu \mathrm{m})$ were pre-treated with fresh xylene for 10 min and rehydrated using gradient alcohol. The sections were microwaved at high power for $25 \mathrm{~min}$ in citrate buffer, $\mathrm{pH}$ 6.0, allowed to cool and then washed in phosphate-buffered saline. The slides were examined independently by two of the authors, without any information pertaining to the patients or the results of the routine histological examination. The Ki-67-LI, Smac and VEGF expression in 40 pre-treatment rectal tumor biopsies and operative specimens was analyzed by immunohistochemistry using specific antibodies. The PV-9000 polymer detection system for immunohistological staining was purchased from Beijing Zhongshan Golden Bridge Biotechnology Company (China). The test was performed when necessary. The slides were evaluated for this study by an expert pathologist.

Methods of analysis. Sections were analyzed at a total magnification of $x 400$. Each core was assigned a continuous score of percentage positivity, representative of the approximate area of immunostaining. For Smac, positive staining was defined as plasma which stained light yellow or pale brown in the section. According to the percentage of positive cells, samples with $<5 \%$ positive cells were defined as negative, samples with $10-30 \%$ positive cells were weakly positive and samples with $>30 \%$ positive cells were characterized as strongly positive. For VEGF, the percentage of cells with positivity in the cytoplasm was determined, and the scoring system was: 0 , negative for VEGF; $1+,<20 \%$ positive cells; $2+$, $20-50 \%$ positive staining and $3+,>50 \%$ staining. For Ki-67, all stained nuclei were scored. Tumor reactivity was expressed as the Ki-67 index (i.e., the number of stained tumor cells per 1,000 cells in each section). The percentage of cells showing distinct nuclear staining was determined.

Statistical analysis. Statistica 13.0 was used for the statistical analysis. Smac, VEGF and Ki-67 expression was evaluated using the Chi-square or Fisher's exact tests. $\mathrm{P}=0.05$ was set as the threshold for statistical significance.

\section{Results}

Expression of Smac, VEGF and Ki-67 in pre-treatment biopsies and post-operative specimens (Fig. 1 and Table I). Tumors were classified into two groups according to the percentage of Smac staining, i.e., high or low expression. Of the 40 pretreatment biopsies, Smac was highly expressed in $24(60 \%)$ specimens. Of the 32 post-operative specimens, Smac was highly expressed in 15 (46.9\%) cases. Smac expression was 

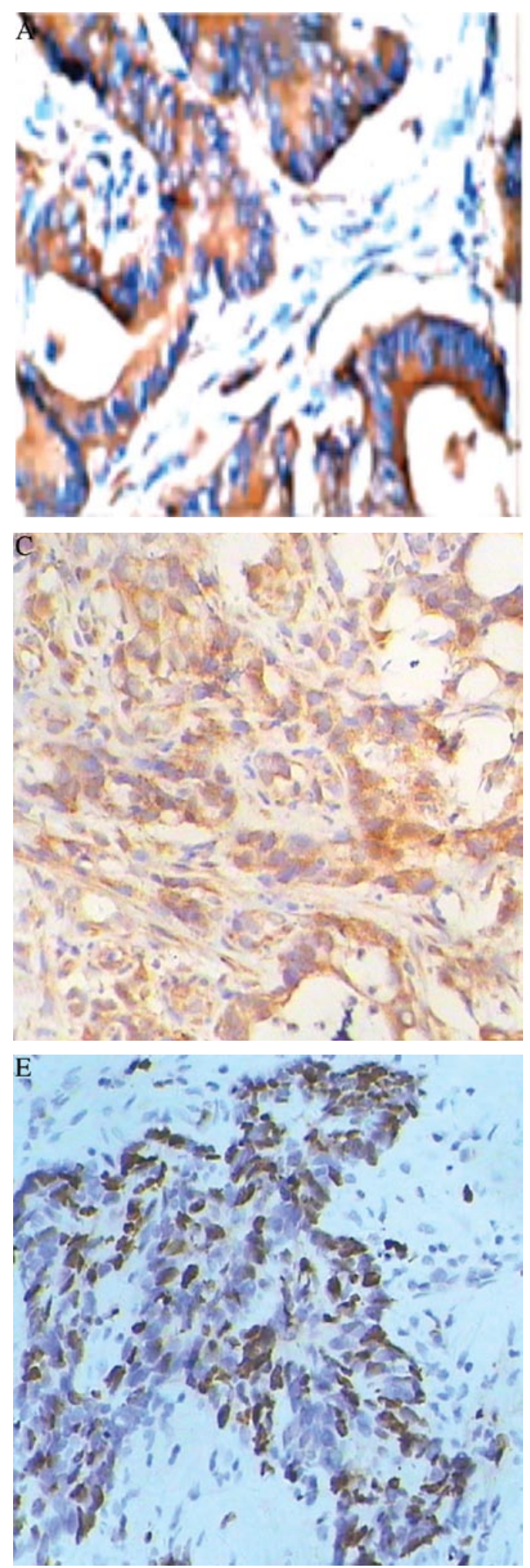
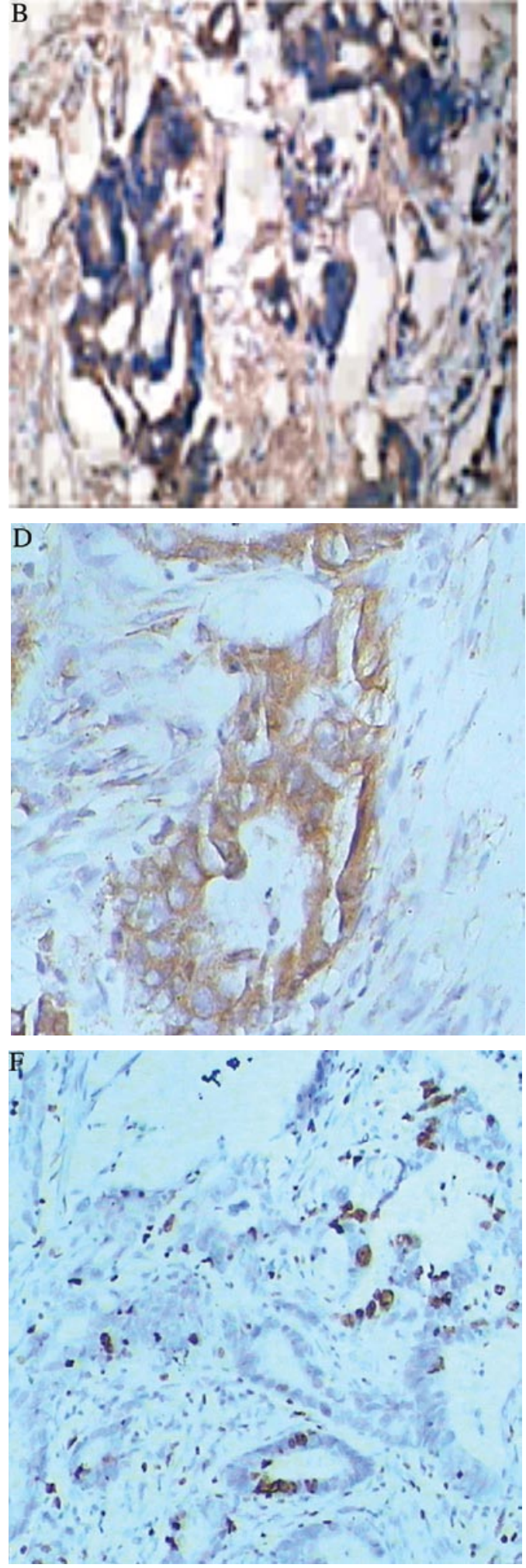

Figure 1. Smac, VEGF and Ki-67 expression. (A) Smac expression in a pre-treatment biopsy, 80\%; (B) Smac expression in a surgical specimen, $15 \%$; (C) VEGF expression in a pre-treatment biopsy, +; (D) VEGF expression in a surgical specimen, +++; (E) Ki-67 expression in a pre-treatment biopsy, 75\%; (F) Ki-67 expression in a surgical specimen, 5\%.

slightly lower after neoadjuvant therapy ( $\mathrm{p}=0.044)$. The VEGFstained signal was located in the cytoplasm and was present as a bulky yellow grain. Of the pre-treatment biopsies and postoperative specimens, $23(57.5 \%)$ and $32(80 \%)$, respectively, were positive. This difference was statistically significant $(\mathrm{p}=0.030$ ). Tumors were classified into two groups according to the percentage of $\mathrm{Ki}-67$ staining and were characterized as exhibiting high or low proliferation. Tumors were determined to be highly proliferative when $>25 \%$ of cells were $\mathrm{Ki}-67$ positive. Of the 40 pre-treatment biopsies and post-operative specimens, Ki-67 was highly expressed in $20(50 \%)$ and $17(42.5 \%)$ cases, respectively. Ki-67 expression was slightly 
Table II. Correlations between Smac,VEGF and Ki-67 expression and clinicopathological characteristics.

\begin{tabular}{|c|c|c|c|c|c|c|c|c|c|c|}
\hline \multirow[b]{2}{*}{$\begin{array}{l}\text { Clinicopathological } \\
\text { characteristics }\end{array}$} & \multirow[b]{2}{*}{$\begin{array}{l}\text { No. of } \\
\text { cases }\end{array}$} & \multicolumn{3}{|c|}{ Smac } & \multicolumn{3}{|c|}{ VEGF } & \multicolumn{3}{|c|}{ Ki-67 } \\
\hline & & Low & High & P-value & Negative & Positive & P-value & $\begin{array}{c}\text { Low } \\
(\leq 10 \%)\end{array}$ & $\begin{array}{l}\text { High } \\
(>10 \%)\end{array}$ & P-value \\
\hline \multicolumn{11}{|l|}{ Age (years) } \\
\hline$<50$ & 16 & 5 & 11 & 0.356 & 7 & 9 & 0.896 & 7 & 9 & 0.519 \\
\hline$\geq 50$ & 24 & 11 & 13 & & 10 & 14 & & 13 & 11 & \\
\hline \multicolumn{11}{|l|}{ Gender } \\
\hline Male & 28 & 10 & 18 & 0.490 & 12 & 16 & 0.944 & 13 & 15 & 0.490 \\
\hline Female & 12 & 6 & 6 & & 5 & 7 & & 7 & 5 & \\
\hline \multicolumn{11}{|l|}{ Tumor size $(\mathrm{cm})$} \\
\hline$\leq 5$ & 30 & 11 & 19 & 0.482 & 13 & 17 & 1.000 & 17 & 13 & 0.144 \\
\hline$>5$ & 10 & 5 & 5 & & 4 & 6 & & 3 & 7 & \\
\hline \multicolumn{11}{|l|}{ Adenocarcinoma } \\
\hline $\begin{array}{l}\text { Well- and moderately } \\
\text { differentiated }\end{array}$ & 28 & 11 & 17 & 1.000 & 13 & 15 & 0.443 & 16 & 12 & 0.168 \\
\hline Poorly differentiated & 12 & 5 & 7 & & 4 & 8 & & 4 & 8 & \\
\hline \multicolumn{11}{|l|}{ Lymph nodal status } \\
\hline Present & 16 & 10 & 6 & 0.018 & 3 & 13 & 0.013 & 4 & 12 & 0.010 \\
\hline Absent & 24 & 6 & 18 & & 14 & 10 & & 16 & 8 & \\
\hline \multicolumn{11}{|l|}{ Infiltration depth } \\
\hline $\mathrm{T} 2$ & 13 & 3 & 10 & 0.130 & 9 & 4 & 0.018 & 8 & 5 & 0.311 \\
\hline $\mathrm{T} 3$ & 27 & 13 & 14 & & 8 & 19 & & 12 & 15 & \\
\hline \multicolumn{11}{|l|}{ TNM stage } \\
\hline II & 23 & 6 & 17 & 0.037 & 13 & 10 & 0.037 & 13 & 10 & 0.337 \\
\hline III & 17 & 10 & 7 & & 4 & 13 & & 7 & 10 & \\
\hline
\end{tabular}

lower after neoadjuvant therapy, but no statistical significance was found. No considerable differences in immunoreactivity were noted between the core and invasive edge of the tumors.

Correlations between Smac, VEGF and Ki-67 expression of the pre-treatment biopsies and patient clinicopathological characteristics (Table II). The level of Smac expression was significantly correlated with lymph node metastasis $(\mathrm{p}=0.018)$ and TNM stage $(\mathrm{p}=0.037)$, but not with histological type, depth of tumor invasion, tumor size, age and gender of the patients $(p>0.05)$. VEGF expression exhibited no statistical significance among the different histological types of rectal cancer $(\mathrm{p}=0.443)$. A significant relationship was noted between patients with and without lymph nodal metastasis $(\mathrm{p}=0.013)$. It was significantly correlated with the depth of tumor invasion $(\mathrm{p}=0.018)$ and TNM stage $(\mathrm{p}=0.037)$. No significant correlation was noted between VEGF expression and gender, age and tumor size ( $p>0.05)$. Ki-67 expression was statistically significant between patients with and without lymph nodal metastasis $(\mathrm{p}=0.010)$. The remaining variables that were evaluated (age, gender, TNM stage, tumor size, histological type and depth of tumor invasion) were not significantly associated with $\mathrm{Ki}-67$ expression $(\mathrm{p}>0.05)$.

Correlations between predictive markers and tumor response (Table III). Smac, VEGF and Ki-67 expression was signifi- cantly correlated with tumor reponse to neoadjuvant therapy ( $\mathrm{p}=0.011,=0.010$ and $=0.034$, respectively). Tumors with a high expression of Smac and a low expression of Ki-67 or VEGF were more sensitive to neoadjuvant therapy.

Correlations between Smac and VEGF and Ki-67 expression (Table IV). A significant correlation was noted between Smac and $\mathrm{Ki}-67$ expression $(\mathrm{p}=0.010)$. However, no correlation was found between VEGF and Ki-67 expression ( $p>0.05)$.

\section{Discussion}

Although beneficial results are obtained with the current multimodal treatment of rectal cancer, 'patient-tailored' treatments are expected to provide greater benefit. This study analyzed the potential value of Smac, VEGF and Ki-67 proteins in predicting the response of tumors to pre-operative chemoradiation.

Second mitochondria-derived activator of caspase/direct inhibitor of apoptosis-binding protein with a low pI (Smac/ DIABLO) is a recently identified pro-apoptotic protein that interacts with and inhibits numerous IAPs, including survivin $(11,22)$. Smac binds to the domain of X-linked inhibitor of apoptosis (XIAP) which is responsible for binding to processed caspase-9, thus, antagonizing the anti-apoptotic function of XIAP. Evidence suggests that the pro-apoptotic function of Smac/DIABLO is linked to an additional mechanism that is 
Table III. Correlations between predictive markers and tumor response.

\begin{tabular}{|c|c|c|c|c|c|c|}
\hline \multirow[b]{2}{*}{ Predictive markers } & \multirow[b]{2}{*}{ No. of cases } & \multicolumn{3}{|c|}{ Tumor response } & \multirow[t]{2}{*}{$\chi^{2}$-value } & \multirow[t]{2}{*}{$\mathrm{P}$-value } \\
\hline & & $\mathrm{CR}$ & PR & NR & & \\
\hline Smac & & & & & 8.985 & 0.011 \\
\hline Low & 16 & 2 & 4 & 10 & & \\
\hline High & 24 & 5 & 15 & 4 & & \\
\hline VEGF & & & & & 9.241 & 0.010 \\
\hline Negative & 17 & 7 & 9 & 1 & & \\
\hline Positive & 23 & 3 & 9 & 11 & & \\
\hline Ki-67-LI & & & & & 6.781 & 0.034 \\
\hline Low & 20 & 7 & 11 & 2 & & \\
\hline High & 20 & 6 & 5 & 9 & & \\
\hline
\end{tabular}

CR, complete response; PR, partial response; NR, no response; Ki-67-LI, Ki-67 labeling index.

Table IV. Correlations between Smac and VEGF and Ki-67 expression.

\begin{tabular}{|c|c|c|c|c|c|c|c|c|}
\hline & \multicolumn{4}{|c|}{ Smac } & \multicolumn{4}{|c|}{ VEGF } \\
\hline & Low & High & $\chi^{2}$-value & P-value & Negative & Positive & $\chi^{2}$-value & P-value \\
\hline Ki-67 & & & 6.667 & 0.010 & & & 0.921 & 0.337 \\
\hline Low & 4 & 16 & & & 10 & 10 & & \\
\hline High & 12 & 8 & & & 7 & 13 & & \\
\hline Smac & & & & & & & 0.614 & 0.443 \\
\hline Low & & & & & 8 & 8 & & \\
\hline High & & & & & 9 & 15 & & \\
\hline
\end{tabular}

different from IAP binding (23). The role of this protein during carcinogenesis has yet to be investigated. However, Smac/ DIABLO mRNA levels have been found to be significantly lower in lung cancers when compared to normal tissues. It was shown that patients with low Smac/DIABLO mRNA levels had worse prognosis. The presence of mature Smac was not sufficient to trigger the apoptosis of healthy cells. However, cells with excess Smac protein were greatly sensitized to apoptotic triggers, such as etoposide exposure (24).

We initially reported the correlation between Smac expression and tumor response to pre-operative chemoradiation. In the present study, the level of Smac expression was significantly correlated with lymph node metastasis and TNM stage $(\mathrm{p}<0.05)$. It was noted that tumors with a high Smac expression were more sensitive to neoadjuvant therapy. Zheng et al found that the stable transfer of the extrinsic Smac gene and its overexpression in cervical cancer cell lines significantly enhanced the expression and activities of cellular caspase- 3 and ameliorated the apoptosis-inducing effects of irradiation on cancer cells, a novel strategy in the improvement of radiotherapeutic effects on cervical cancer (25). Jia et al demonstrated that mature Smac enhanced the susceptibility of K562 and CEM cells to TRAIL-induced apoptosis (26). Overexpression of the mature Smac protein also inhibited proliferation, as detected by the reduced colony formation and $\mathrm{Ki}-67$ expression in leukaemic cells. Cell cycle analysis showed that Smac transfectants displayed significant G0/G1 arrest and reduction in 5-bromo-20-deoxyuridine incorporation. In addition, Smac was found to sensitize human acute myeloid leukaemia blasts to the cytochrome c-induced activation of caspase-3. Thus, it was shown that Smac/DIABLO exhibits a potential role in increasing apoptosis and suppressing proliferation in human leukaemic cells (26). Additionally, a significant negative correlation between Smac and Ki-67 expression $(\mathrm{p}<0.05)$ was found.

We investigated the level of VEGF expression in 40 pretreatment biopsies and post-operative specimens of rectal cancer patients. The patient group with lymph nodal metastasis exhibited a higher expression compared to the group without matestasis. VEGF expression was also elevated with increasing depth of invasion. VEGF expression was temporarily overexpressed following neoadjuvant therapy. Zlobec et al investigated the relationship of VEGF expression of rectal cancer patients and neoadjuvant therapy. Low or absent VEGF in pre-irradiation rectal tumor biopsies was strongly associated with complete tumor response. By contrast, non-response tumors were more highly immunoreactive and had a higher VEGF expression than completely responsive tumors (27). Giralt et al studied 81 patients with locally advanced rectal cancer treated with pre-operative radiotherapy and found that the VEGF expression was associated with a 
less favorable disease-free survival. This difference was significantly related to the development of metastatic disease. A high expression of VEGF was associated with poor prognosis (28). VEGF, assessed immunohistochemically from pre-treatment tumor biopsies, may be a useful marker in the prediction of tumor response to pre-operative CRT (29). Few reports exist, however, involving VEGF expression in rectal cancer patients both prior to treatment and post-treatment. In the present study, the VEGF expression increased significantly following neoadjuvant therapy at 4-6 weeks. In a previous study, Inoue et al investigated the interactions between CRT and the expression of VEGF in vivo and in vitro. The study showed that there was a significant increase in local VEGF levels, in vivo and in vitro, after CRT, particularly in viable cancer cells, possibly due to a reduction in tumor volume induced by the CRT (30).

We aimed to correlate the expression of Ki-67 with response in a patient population with locally advanced rectal tumors. Ki-67 expression was clearly diminished following pre-operative CRT. Findings by Rau et al found the downregulation of $\mathrm{Ki}-67$ after neoadjuvant treatment, indicating that highly proliferative tumor cells are the most sensitive to neoadjuvant treatment (31). However, our results showed that patients with a favorable response during pre-operative CRT exhibited a low expression of $\mathrm{Ki}-67$ in the resection specimens, suggesting that the effect of the treatment on proliferation was crucial for a beneficial response. Kim et al observed a significantly positive correlation between the cellular proliferative index and tumor response in rectal cancer after pre-operative concurrent chemoradiotherapy (CCRT), indicating that Ki-67 labeling may be a useful parameter for radiosensitive tumors selected for CCRT (32). Scholzen et al investigated a large number of cancer samples and reported that the value of $\mathrm{Ki}-67$ as a prognostic marker for survival and tumor recurrence was repeatedly confirmed in uni- and multivariate analyses (33). There are a number of indications that $\mathrm{Ki}-67$ protein expression is a requirement for progression through cell division. $\mathrm{Ki}-67$ expression is highly associated with cell proliferation. Adell et al studied 152 rectal cancers and found that Ki-67 expression of pre-operative biopsies correlated with radiotherapy sensitivity (34). The interaction between Ki-67 status and the benefit of radiotherapy was significant for a reduced recurrence rate. Many Ki-67-stained tumor cells in the preoperative biopsy predicted an increased treatment failure rate after radiotherapy of rectal cancer. Debucquoy et al found that $\mathrm{Ki}-67$ was present in all biopsies and resection specimens and was down-regulated after therapy in $83 \%$ of the patients (35). In this study, the expression decreased significantly with a median value of $90 \%$ in the biopsies compared to $45 \%$ in the resection specimens. A lower pre-therapy concentration of Ki-67 was associated with CR. Furthermore, no significant correlation was found between $\mathrm{Ki}-67$ expression and patient gender, age and tumor size, while Ki-67 was significantly correlated with lymph node metastasis, pathological stage and prognosis. On the other hand, a statistically significant difference $(\mathrm{p}<0.05)$ in the $\mathrm{Ki}-67$ expression between patients with and without lymph nodal metastasis was noted in our study. Patients with a high expression of Ki-67 presented with lymph nodal metastasis and exhibited a poor prognosis. These results indicated that $\mathrm{Ki}-67$ expression correlated with tumor progression. No statistical significance was found between Ki-67 expression and age, gender, histological type, tumor size and the depth of tumor invasion. Ki-67 expression was higher in the patient group with lymph nodal metastasis and tumor-eroded chorion group than the negative group. The Ki-67 expression decreased after the patients received CRT, indicating that $\mathrm{Ki}-67$ expression was closely related to a deteriorating degree of tumor and biology behavior. No statistical significance between Ki-67 expression and tumor differentiation was noted in our study, which may have been due to the small sample size. In addition, tumor cell proliferation may be influenced by various factors. The degree of malignancy of tumors is related to the invasive ability of tumor cells. On the other hand, different methods and diverse samples result in varied conclusions. To ensure the significance of Ki-67 expression in rectal cancer, studies with a large population of samples are required. As concurrent radiotherapy and chemotherapy enhance the efficacy of therapy, we suggest that this type of treatment is applied to patients with rectal cancer, even in those with a high expression of Ki-67.

The limitations of our study included: i) The investigation comprised a small patient population; ii) the classification of clinical stages using magnetic resonance imaging for rectal cancer is not exact; and iii) the protein expression in biopsies may not be representative of the entire tumor.

In conclusion, the results of this study indicate that Smac, VEGF and Ki-67, assessed immunohistochemically from pre-treatment tumor biopsies, may be useful markers in the prediction of tumor response to pre-operative CRT. Through the assessment of Smac, VEGF and Ki-67 expression, we can also estimate the degree of malignancy and prognosis of rectal cancer patients.

\section{Acknowledgements}

This study was supported by Shandong Tumor Hospital, China.

\section{References}

1. Sauer R, Becker H, Hohenberger W, et al: Preoperative versus postoperative chemoradiotherapy for rectal cancer. N Engl J Med 351: 1731-1740, 2004.

2. Bosset JF, Calais G, Mineur L, et al: Enhanced tumoricidal effect of chemotherapy with preoperative radiotherapy for rectal cancer: preliminary results-EORTC 22921. J Clin Oncol 23: 5620-5627, 2005

3. Bosset JF, Collette L, Calais G, et al: Chemotherapy with preoperative radiotherapy in rectal cancer. $\mathrm{N}$ Engl J Med 355: 1114-1123, 2006.

4. Gerard J, Romestaing P, Bonnetain F, et al: Preoperative chemoradiotherapy (CT-RT) improves local control in T3-4 rectal cancers: results of the FFCD 9203 randomised trial. Int J Radiat Oncol Biol Phys 63: 2-3, 2005.

5. Das $\mathrm{P}$ and Crane $\mathrm{CH}$ : Preoperative and adjuvant treatment of localized rectal cancer. Curr Oncol Rep 8: 167-173, 2006.

6. McLean CM and Duncan W: Rectal cancer: a review of randomized trials of adjuvant radiotherapy. Clin Oncol 7: 349-358, 1995.

7. Friedmann P, Garb JL, Park WC, et al: Pace OT survival following moderate-dose preoperative radiation therapy for carcinoma of the rectum. Cancer 55: 967-973, 1985.

8. Kerman HD, Roberson SH, Bloom TS, et al: Rectal carcinoma. Long-term experience with moderately high-dose preoperative radiation and low anterior resection. Cancer 69: 2813-2819, 1992. 
9. Kim DW, Lim SB, Kim DY, et al: Pre-operative chemo-radiotherapy improves the sphincter preservation rate in patients with rectal cancer located within $3 \mathrm{~cm}$ of the anal verge. Eur J Surg Oncol 32: 162-167, 2006.

10. Minsky BD: Oxaliplatin-based combined-modality therapy for rectal cancer. Semin Oncol 30: 26-33, 2003.

11. Du C, Fang M, Li Y, Li L and Wang X: Smac, a mitochondrial protein that promotes cytochrome $\mathrm{c}$ dependent caspase activation by elimination IAP inhibition. Cell 102: 33-42, 2000.

12. Kang S-M, Maeda K, Onoda N, et al: Combined analysis of p53 and vascular endothelial growth factor in CRC for determination of tumor vascularity and liver metastasis. Int J Cancer 74 502-507, 1997.

13. Wong MP, Cheung N, Yuen ST, Leung SY and Chung LP: Vascular endothelial GF is up-regulated in the early pre-malignant stage of colorectal tumor progression. Int J Cancer 81: 845-850, 1999.

14. Tonini T, Rossi F and Claudio PP: Molecular basis of angiogenesis and cancer. Oncogene 22: 6549-6556, 2003.

15. Lutsenko SV, Kiselev SM and Severin SE: Molecular mechanisms of tumor angiogenesis. Biochem 68: 286-300, 2003.

16. Ferrara N, Gerber H-P and LeCouter J: The biology of VEGF and its receptors. Nat Med 9: 669-676, 2003.

17. Falm E: Angiogenesis inhibitors in clinical development; where are we now and where are we going? Br J Cancer 90: 1-7, 2004.

18. Ferrara N: Vascular endothelial growth factor: basic science and clinical progress. Endocr Rev 25: 581-611, 2004.

19. Harmey JH and Bouchier-Hayes D: Vascular endothelial growth factor (VEGF), a survival factor for tumour cells: implications for anti-angiogenic therapy. Bioessays 24: 280-283, 2002.

20. Willett C, Boucher Y, di Tomaso E, et al: Direct evidence that the VEGF-specific antibody bevacizumab has anti-vascular effects in human rectal cancer. Nat Med 10: 145-147, 2004.

21. Gerdes J, Lemke H, Baisch H, Wacker HH, Schwab U and Stein H: Cell cycle analysis of a cell proliferation-associated human nuclear antigen defined by the monoclonal antibody Ki-67. J Immunol 133: 1710-1715, 1984.

22. Song Z, Yao X and Wu M: Direct interaction between survivin and Smac/DIABLO is essential for the anti-apoptotic activity of survivin during taxol-induced apoptosis. J Biol Chem 278 23130-23140, 2003.

23. Roberts DL, Merrison W, MacFarlane M and Cohen GM: The inhibitor of apoptosis protein-binding domain of Smac is not essential for its proapoptotic activity. J Cell Biol 153: 221-228, 2001.
24. Sekimura A, Konishi A, Mizuno K, et al: Expression of Smac/ DIABLO is a novel prognostic marker in lung cancer. Oncol Rep 11: 797-802, 2004

25. Zheng LD, Xiong ZF, Zhu JW and Wang ZH: Effects of Smac gene over-expression on the radiotherapeutic sensitivities of cervical cancer cell line HeLa [J]. Chin Med J 118: 226-230, 2005.

26. Jia L, Patwari Y, Kelsey SM, et al: Role of Smac in human leukaemic cell apoptosis and proliferation. Oncogene 22: 1589-1589, 2003.

27. Zlobec I, Steele R and Compton CC: VEGF as a predictive marker of rectal tumor response to preoperative radiotherapy. Cancer 104: 2517-2521, 2005.

28. Giralt J, Navalpotro B, Hermosilla E, et al: Prognostic significance of vascular endothelial growth factor and cyclooxygenase- 2 in patients with rectal cancer treated with preoperative radiotherapy. Oncology 71: 312-319, 2006.

29. Kakeji Y, Koga T, Sumiyoshi Y, et al: Clinical significance of vascular endothelial growth factor expression in gastric cancer. $\mathrm{J}$ Exp Clin Cancer Res 21: 125-129, 2002.

30. Inoue $\mathrm{Y}$, Ojima E, Watanabe $\mathrm{H}$, et al: Does preoperative chemoradiotherapy enhance the expression of vascular endothelial growth factor in patients with rectal cancer? Oncol Rep 18: 369-375, 2007.

31. Rau B, Sturm I, Lage H, et al: Dynamic expression profile of p21WAF1/CIP1 and Ki-67 predicts survival in rectal carcinoma treated with preoperative radiochemotherapy. J Clin Oncol 21: 3391-3401, 2003

32. Kim NK, Park JK, Lee KY, et al: p53, BCL-2 and Ki-67 expression according to tumor response after concurrent chemoradiotherapy for advanced rectal cancer. Ann Surg Oncol 8: 418-424, 2001.

33. Scholzen T and Gerdes J: The Ki-67 protein: from the known and the unknown. J Cell Physiol 182: 311-322, 2000.

34. Adell G, Zhang H, Jansson A, Sun XF, Stål O and Nordenskjöld B Decreased tumor cell proliferation as an indicator of the effect of preoperative radiotherapy of rectal cancer Int J Radiat Oncol Biol Phys 50: 659-663, 2001.

35. Debucquoy A, Goethals L, Geboes K, Roels S, Mc Bride WH and Haustermans K: Molecular responses of rectal cancer to preoperative chemoradiation. Radiother Oncol 80: 172-177, 2006. 